\title{
High-speed deformation and fracture of the dioxide-zirconium ceramics and zirconium alumina concrete
}

\author{
A. Bragov ${ }^{1}$, L. Kruszka², A. Lomunov³ ${ }^{3}$ A. Konstantinov ${ }^{1}$, D. Lamzin ${ }^{3}$, and A. Filippov ${ }^{1}$ \\ ${ }^{1}$ Research Institute of Mechanics, State University of Nizhny Novgorod, Russia \\ ${ }^{2}$ Military University of Technology, Warsaw, Poland \\ ${ }^{3}$ State University of Architecture and Civil Engineering, Nizhny Novgorod, Russia
}

\begin{abstract}
The results of dynamic tests of two refractory materials based on zirconium dioxide: three types of ceramics and barium-aluminate concrete. The ceramic specimens had various initial density and porosity and also differed by technology of manufacturing. Compression tests were carried out using the Kolsky technique and its updating - specimen in a rigid jacket. The dynamic stress-strain curves were obtained. The strong influence of sample's initial grain composition, mode of stressed-strained state and technologies of manufacturing of specimens onto ceramics mechanical properties is marked.
\end{abstract}

\section{Introduction}

In the event of a serious accident in a nuclear reactor with its core melting, it becomes crucial to prevent the propagation of the resulting radioactive flux into the environment. The problem of confining the melting core is vital for all the countries using nuclear power plant. A corium trap material is anticipated to undergo an influence of various factors, such as mechanic and thermal shocks resulting after falling the melt and the structural elements into the trap, a vapor explosion resulting from the contact of the melt with water, chemical interaction with corium, and a radiation effect. The anticipated high temperature of corium $(2800-3000 \mathrm{~K})$ and its chemical activity in oxidizing conditions considerably reduce the choice of materials that could be used for the trap.

The currently developed corium trap based on using the highly refractory dioxide zirconium concrete has to increase the life of a protecting trap by two orders of magnitude as compared to the construction concrete. However, use of the cements with the melting temperatures of 2300 $\mathrm{K}$ and lower in the above concrete reduces the merits of the refractory component - zirconium dioxide.

The dioxide zirconium-based sintered ceramics is free from the above drawbacks.

To assess the potential of using dioxide zirconium ceramics, comparative studies of dynamic strength of the ceramics and of some highly refractory concretes under high-velocity loading are to be conducted. The aim of the paper is to analyze experimentally the influence of the loading rate on the strength properties of dioxidezirconium ceramics and concrete based on zirconium aluminate.

\section{Tested materials}

Specimens of zirconium-dioxide ceramics (ZDC) of three batches were made differing in initial density, porosity and grain composition (Table 1). The specimen production technology was close to the industrial one. It included grinding to obtain the required fractional composition, magnetic and chemical purification, introduction of temporary plasticizer, compaction (pressure of $100 \mathrm{MPa}$ ) and annealing ( $2000 \mathrm{~K}$ for 13 hours).

Also the samples of zirconium alumina concrete (ZAC) were subjected to tests. The zirconium ceramics of three batches and the ZAC concrete have the following properties under ambient conditions (Table 2):

The ZAC specimens were made of the electrically melted zirconium dioxide, stabilized by yttrium oxide ( $87 \%$ of $\mathrm{ZrO}_{2}+10 \%$ of $\mathrm{Y}_{2} \mathrm{O}_{3}$ ), as the filler and the bariumaluminate cement as the binder (mass fraction 6\%).

The tested specimens were manufactured in the form of tablets about $20 \mathrm{~mm}$ in diameter and $10 \mathrm{~mm}$ in thickness. The geometry of the specimens was chosen according to Davies-Hunter criteria [1] to minimize inertia and friction effects.

\section{Test methods}

To obtain dynamic properties of tested materials the experimental apparatus using the traditional [2] and modified $[3,4]$ (specimen in a rigid jacket) SHPB scheme was used. The first method is allowed to obtain the compressive dynamic diagrams $\sigma \sim \varepsilon$ at 1D stress state whereas the second one - at volumetric stress state and uniaxial strain. During the modified tests a specimen is located inside the rigid steel jacket that confines its radial strain. Alongside with the deformation diagrams, studying the shear resistance (one of the basic mechanical characteristics of ceramics) also presents great interest. Determining above characteristic as a function of strain rate $\dot{\varepsilon}$ or loading rate $\dot{\sigma}$ or pressure $P$ plays an important role in formulating the equations of state for ceramics and other porous medias.

Volumetric compressive stress in specimen is realized by its location between end-faces of pressure bars inside a rigid steel of duralumin jacket that confines radial strain of specimen [3]. In these experiments longitudinal stress $\sigma_{x}$ and strain $\varepsilon_{x}$ are obtained by the traditional SHPB relationships. Radial stress $\sigma_{r}$ is determined by registration of peripheral elastic strain of the jacket equipped by strain gages [4]. Two components of the stress tensor $\sigma_{x}$ and $\sigma_{r}$ 
Table 1. Particle-size composition of ceramics from the three batches ( $\%$ of weight).

\begin{tabular}{|l|c|c|c|c|c|c|}
\hline \multirow{2}{*}{$\begin{array}{l}\text { Batch } \\
\text { No }\end{array}$} & \multicolumn{7}{|c|}{ Fractional Sizes, mm } \\
\cline { 2 - 7 } & $<\mathbf{0 . 0 5}$ & $<\mathbf{0 . 2}$ & $\mathbf{0 . 2 - 0 . 3 1 5}$ & $\mathbf{0 . 3 1 5 - 0 . 4}$ & $\mathbf{0 . 6 3 - 1}$ & $\mathbf{1 - 2}$ \\
\hline 1 & 40 & & 20 & & 40 & \\
2 & 25 & & 25 & 20 & & 30 \\
3 & $15^{*}$ & 30 & & & & 55 \\
\hline
\end{tabular}

* - tetragonal phase. The rest of the fractions were cubical structures stabilized with $\mathrm{Y}_{2} \mathrm{O}_{3}$ with the molar fraction of $10-11 \%$.

allow to determine pressure $P$, volumetric strain $\theta$, shear resistance $\tau$, stress $\sigma_{i}$ and strain $\varepsilon_{i}$ intensity, the factor $\xi$ of lateral pressure (thrust), and, therefore, to construct a set of functions: $\sigma_{x} \sim \varepsilon_{x}, \sigma_{i} \sim \varepsilon_{i}, P \sim \theta, \tau \sim P, \xi \sim P$, etc.

For investigation of tension strength of materials the well known "Brazilian test" was used [5].

The changeable sets of pressure bars are made from high-strength steel or from hard aluminium alloy.

Before placing a sample on-position a few layers of thin $(10 \mu \mathrm{m})$ fluoroplastic film were placed between the end faces of pressure bars and the specimen to reduce friction and to improve the acoustic contact. In jacketconfined tests the gap between the bars and the jacket was also filled with fluoroplastic film and the specimens were tightened up manually. They were tested at ambient temperature.

\section{Results and discussion}

\subsection{Behaviour at uniaxial compressive stress}

Specimens of optimal geometries both of ZDC (three batches) and ZAC were tested under uniaxial compressive stress. By varying striker velocities (i.e. the amplitude of incident pulse) loading modes were selected under which a specimen either retained its apparent integrity and strength or has been destroyed.

Results of testing for ZDC of three batches are summarized in figures 1-3 as stress-strain curves (solid lines). Appropriate strain-rate dependences (dotted lines) are shown in lower part of figures. The corresponding axis is in the right part of these figures.

For each batch of ceramics there are given typical stress-strain curves resulting from averaging of several tests conducted in nominally equal conditions. The lower stress-strain curve (line 1) corresponds to the conditions in which the specimens retain their integrity after the experiment. The upper curve (line 3) was obtained in the conditions of complete failure of the specimens. The intermediate curve (line 2) was obtained for the conditions when the specimens either had little (mainly peripheral) damage or lost their structural cohesion, retaining their apparent integrity.

It is well visible that ceramics batch No 3 has highest strength that probably originates from its lowest porosity as well as the highest static strength (table 2). For all types of ceramics a magnitude of modules of unloading branch is more in 2-3 times than appropriate modules of loading branch of the stress-strain curves for all types of ceramics.

In all figures shown above, the small tables are built-in where for each curve the average strain rates are shown as well as the stress rates calculated as a tangent of an inclination angle of secant module on a site of an active loading of a stress-strain curve.

Similar results were obtained for ZAC specimens.

It is necessary to note, that specimen unloading process for ceramics during a test has very large duration as contrasted with test of metals. The duration of unloading at small level of stress exceeds duration of a loading pulse of in tens time. The reason of this phenomenon can be presence in ceramics a polymeric binding for which there are typically a large coefficient of restitution of the shape and significant nonlinearity of an unloading branch. Owing to a strong local heat-up of a binding matrix during a plastic deformation its viscosity is noticeably incremented and the unloading process goes very slowly. Therefore it is impossible to register full process of ceramic sample unloading and accordingly to ensure the stress-strain curve with full unloading of a sample up to zero stress in it.

\subsection{Investigation of volumetric compressibility}

Results of investigation of volumetric compressibility as well as shear resistance of ZDC (batch No3) are shown below. In figure 4 there are a few curves of volumetric compressibility for ZDC: longitudinal and radial components of stress together with pressure in a specimen vs. strain. All of shown curves are non-linear in character, their loading and unloading branches are differing.

Figure 5 shows the shear strength as a function of pressure $\tau \sim P$ (solid curve). It is visible that this function has linear character. The experimental data processed using the least squares method are approximated by a straight line and can be represented in the form of an equation as: $\tau=C+\operatorname{tg} \varphi \cdot P$, where $C$ and $\varphi$ are conventionally taken to be the adhesion coefficient and the internal friction angle. Such equation is known as the strength condition of Coulomb-More [6].

The lateral thrust ratio (dashed line) in the initial part of specimen loading has great fluctuation because of dividing of one very small value (radial stress) onto another small value (longitudinal stress).

As a result of a serious of tests the curves of shear resistance were constructed and the parameters of the Coulomb-More equation were determined. Factor of lateral pressure $\xi$ is also determined. The results of a number of the executed tests are average and given in the table 3 .

In processing of the experimental information, in addition to average strain rates $\dot{\varepsilon}_{s}$ maximum values of stress rates $\dot{\sigma}_{s}$ in the specimen were determined for each test; these were presented in small build-in tables in figures $1-3$. Figure 6 depicts stress-rate dependence of breaking stresses for each batch of the ceramics and concrete ZAC. Also in figure 6 near axis Y, by using corresponding markers, static compressive strengths of the materials are shown (from Table 2). It is evident that the breaking stresses grow with the stress rate. 
Table 2. Physical-mechanical properties of the tested ceramics and ZAC concrete.

\begin{tabular}{|l|c|c|c|c|}
\hline Property & ZDC Batch No 1 & ZDC Batch No 2 & ZDC Batch No 3 & ZAC concrete \\
\hline Density, g/sm ${ }^{3}$ & $4.7 \div 4.9$ & 4.82 & 4.56 & 4.66 \\
Porosity, \%. & 20 & 17 & 16 & $13-16$ \\
Static Compression Strength, MPa & 39 & 45 & 68 & 35 \\
\hline
\end{tabular}

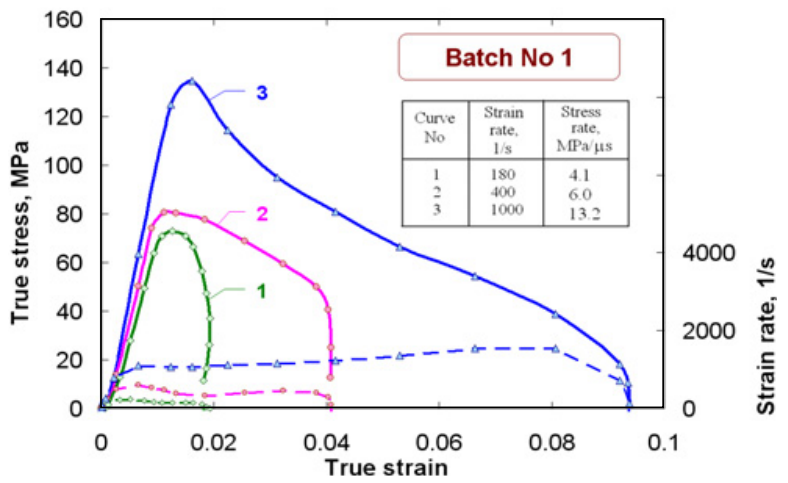

Fig. 1. Behaviour of ZDC batch No 1.

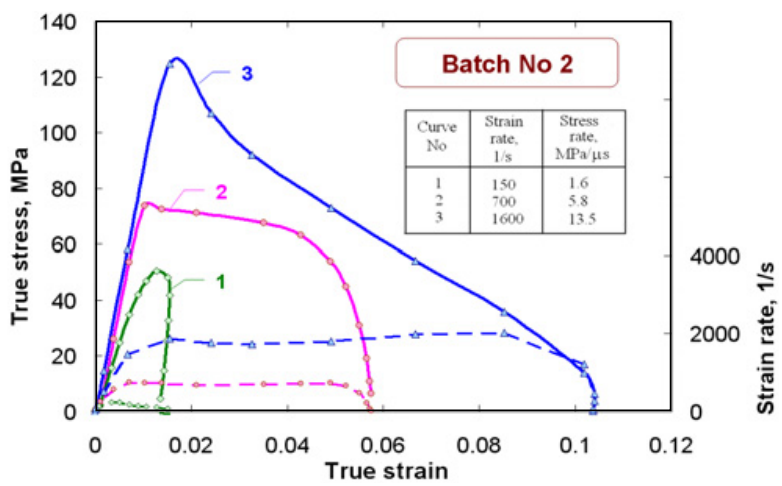

Fig. 2. Behaviour of ZDC batch No 2 .

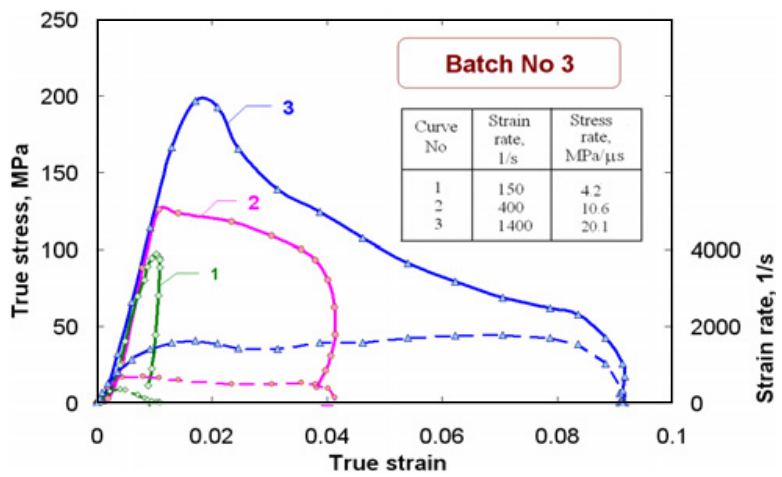

Fig. 3. Behaviour of ZDC batch No 3.

In figure 5 it is well visible, that sites of loading and unloading on curves $\tau \sim P$ and $\xi \sim P$ practically coincide.

It is interesting to compare the dynamic diagrams of a ceramics in similar conditions after tests of specimen in confining jacket and without it (figure 7). The curves 1 and 2 are characteristic for tests in the rigid jacket, and curves 3 - without a jacket. The strain-rate conditions of loading in cases 2 and 3 are similar. It is well visible, that at identical

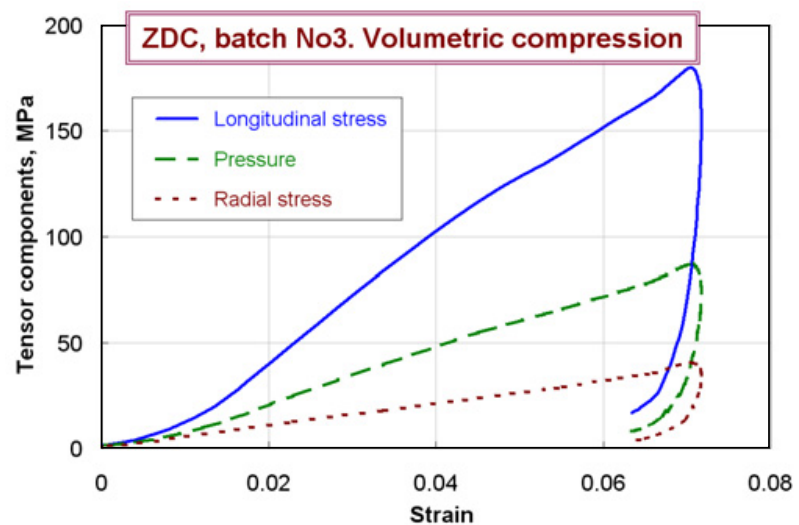

Fig. 4. Volumetric compression of ZDC batch No 3.

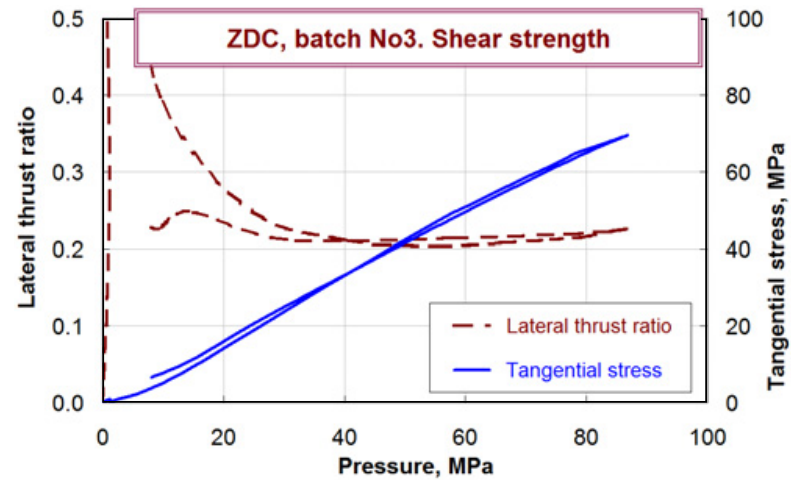

Fig. 5. Shear strength of ZDC batch No 3.

Table 3. Shear resistance for ZDC No 3 and ZAC.

\begin{tabular}{|l|c|c|c|}
\hline Material & $\begin{array}{c}\text { Adhesion } \\
\text { coefficient } \\
C, \mathrm{MPa}\end{array}$ & $\begin{array}{c}\text { Internal } \\
\text { friction angle } \\
\varphi, \text { degree }\end{array}$ & $\begin{array}{c}\text { Lateral } \\
\text { thrust } \\
\text { ratio } \xi\end{array}$ \\
\hline ZDC No3 & 1.37 & 38.7 & 0.224 \\
\hline ZAC & 1.75 & 37.7 & 0.222 \\
\hline
\end{tabular}

strain rate the sample in a jacket maintains considerably large stress, than without it. An abruptness and form of load branches of the diagrams at initial stage in both cases are identical. At excitation of loading impulse of greater amplitude the sample in the jacket maintains accordingly larger stress (curves 1 and 2).

It is well visible, that the specimen tested without a jacket (curve 3) has collapsed at stress $\sim 80 \mathrm{MPa}$, whereas the similar specimens in a rigid jacket (curve 2) have kept integrity at stress more than $300 \mathrm{MPa}$. However, it is necessary to mean, that the sample in a jacket could sustain 


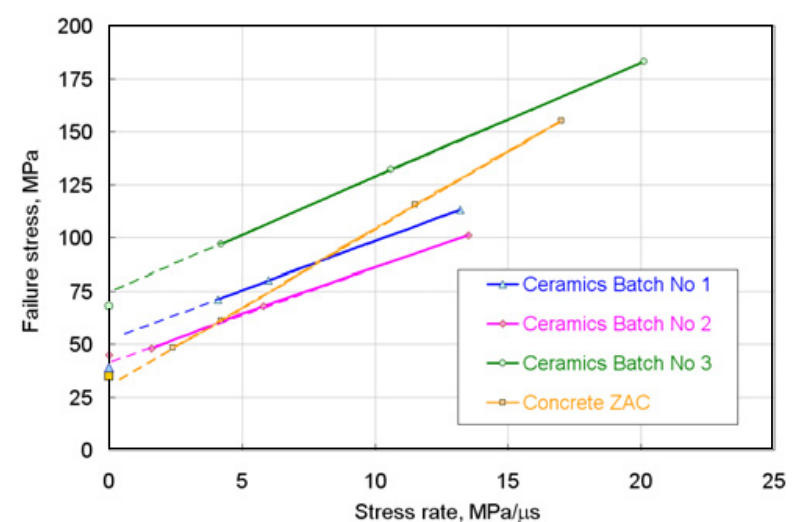

Fig. 6. Failure stress vs. stress-rate.

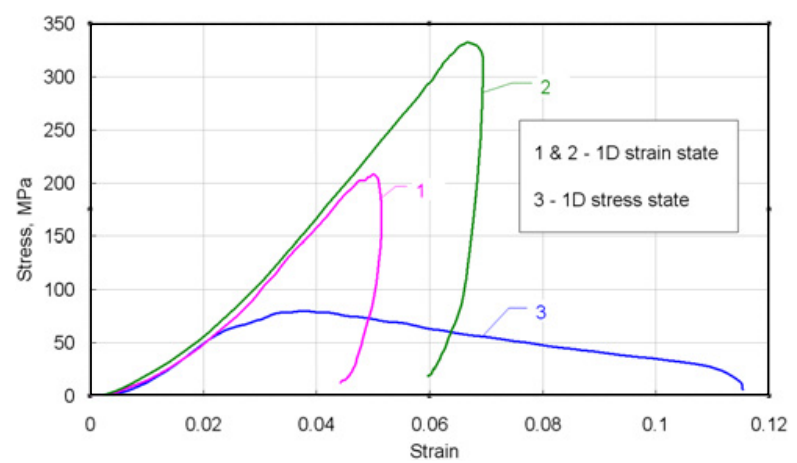

Fig. 7. The influence of stress-strain mode.

without destruction considerably greater stress if to excite in it large amplitude of stress.

\subsection{Dynamic strength at splitting}

The cited above modified Kolsky method for definition of strength of brittle materials by a method of splitting at compression was used for research of properties of the zirconium ceramics of batches No 2 and 3 (Tables 1 and 2) and ZAC concrete. The experimental set-up with pressure bars of a diameter of $20 \mathrm{~mm}$ was used. Specimens of both batches of ceramics as well as ZAC concrete were tested by different geometries (see table 4). During application of load to generatrix of a cylindrical specimen the occurrence of local zones of destruction is possible, caused by localization of the appendix of force and a roughness of a lateral surface of a specimen. For reduction of this effect between the specimen and end faces of pressure bars the damping lining (a thin cardboard) was located.

The amplitude of transmitted pulse allows determining the maximal axial force $P_{\max }$, at which a destruction (splitting) of a sample has been occurred, and further to calculate strength of a material at splitting $\sigma_{t}$. Parametrical dependences of a tensile stress in the specimens vs. time have been obtained. An averaging of these curves by results of a series of tests in similar conditions was carried out. The example of such curves is resulted in figure 8 .

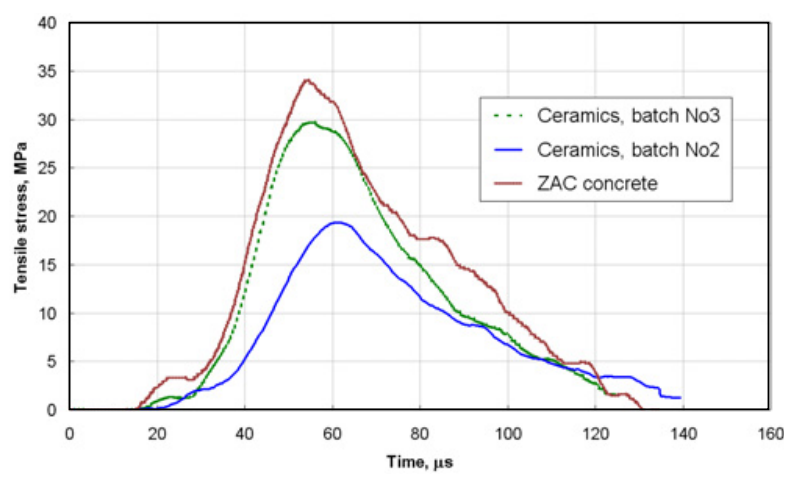

Fig. 8. Failure stress vs. stress-rate.

Table 4. Strength of ZDC and ZAC at splitting.

\begin{tabular}{|l|c|c|c|}
\hline Material & $\begin{array}{c}\text { Length of } \\
\text { a sample, } \mathrm{mm}\end{array}$ & $\begin{array}{c}\text { Diameter of } \\
\text { a sample, } \mathrm{mm}\end{array}$ & $\begin{array}{c}\text { Strength at } \\
\text { splitting, MPa }\end{array}$ \\
\hline ZDC Batch & 9.5 & 20 & 18.9 \\
No 2 & 9.5 & 20 & 16.8 \\
& 19.5 & 20 & 22.5 \\
& 19.5 & 20 & 20.0 \\
\hline ZDC Batch & 10 & 22 & 30.8 \\
No 3 & 10 & 22 & 30.8 \\
& 7 & 22 & 31.1 \\
\hline ZAC & 6.4 & 22 & 29.8 \\
\hline
\end{tabular}

In table 7 this parameter for ZDC of two batches and two sizes of samples everyone as well as for ZAC concrete are given.

It is possible to note, that the strength at splitting poorly depends on the geometrical sizes of samples for both lots of ceramics. The ceramics of batch No2 has a little bit greater disorder of properties, than ceramics of batch No3. The strength of ceramics of batch No3 in $\sim 1.5$ times is higher, than ceramics of batch No2. The ZDC concrete has some greater strength at splitting.

\subsection{The influence of specimen's geometry and condition of loading}

For research of the scale factor as well as effects of inertia and a friction at end-faces of specimens during its radial expansion a few comparative tests of ceramics of three batches were conducted. ZDC specimens have been made with different lengths: batch No $1-2 \mathrm{~mm}, 5 \mathrm{~mm}$ and $10 \mathrm{~mm}$, batch No $2-5 \mathrm{~mm}, 10 \mathrm{~mm}$ and $20 \mathrm{~mm}$, batch No 3 $-5 \mathrm{~mm}$ and $10 \mathrm{~mm}$. Specimens were tested at uniaxial compression. Besides, specimens of ZDC of batch No 1 were tested in a rigid holder.

In figures 9-12 for each batch of ceramics and every length of specimens there are shown as usually two typical stress-strain curves: under condition of preservation of visible integrity of a specimen (or of the minimal its damage - solid lines) and in the condition of full destruction of a specimen (dashed lines). 


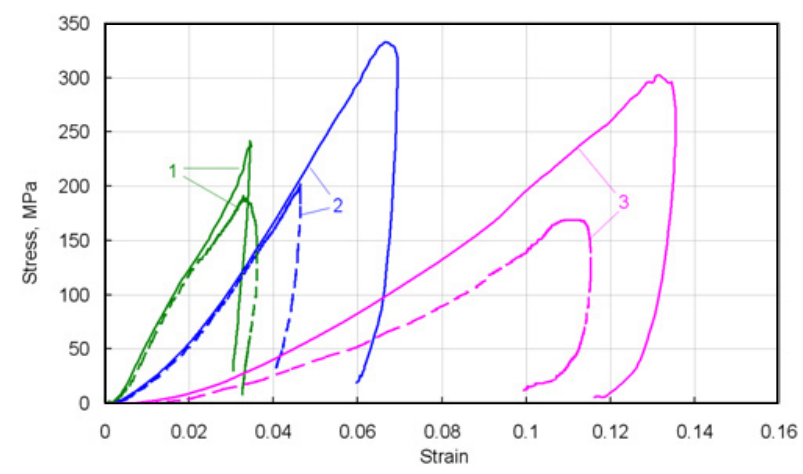

Fig. 9. Behaviour of ZDC No1 at uniaxial strain for different length of specimen: $1-10 \mathrm{~mm}, 2-5 \mathrm{~mm}, 3-2 \mathrm{~mm}$.

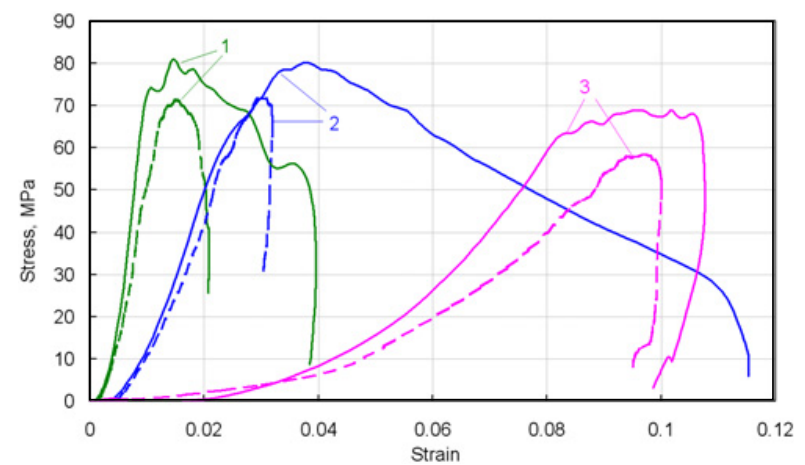

Fig. 10. Behaviour of ZDC No1 at uniaxial stress for different length of specimen: $1-10 \mathrm{~mm}, 2-5 \mathrm{~mm}, 3-2 \mathrm{~mm}$.

It is obvious for all batches of ceramics, that a thickness of specimen has essential influence on a course of the obtained dynamic diagrams: thicker specimens have greater value of modulus of load branch and smaller energy consumption. The strength properties from length of specimen depend feebly.

Well appreciably that the ceramics No3 has higher strength and smaller deformative properties. The values of strength at compression for all types of ceramics poorly depend on thickness of samples, whereas the value of deformation in a point of destruction decreases with growth of thickness of a sample. The average modules of loading branch grow with increase of thickness of a specimen. Such behaviour results to the fact: the specific energy consumption, determined as the area under the curve $\sigma \sim$ $\varepsilon$, for thin samples is much higher, than for thick one.

Value of unloading module of the stress-strain curves is essential more than appropriate module of loading branches.

\section{Conclusions}

The wide spectrum of tests on specimens of zirconium dioxide ceramics allows making the following conclusions.

The carried out experiments of samples of ceramics of various geometry testify to strong influence of specimen thickness onto modules at loading and unloading branch: deformability of thin samples is much higher, than thick.

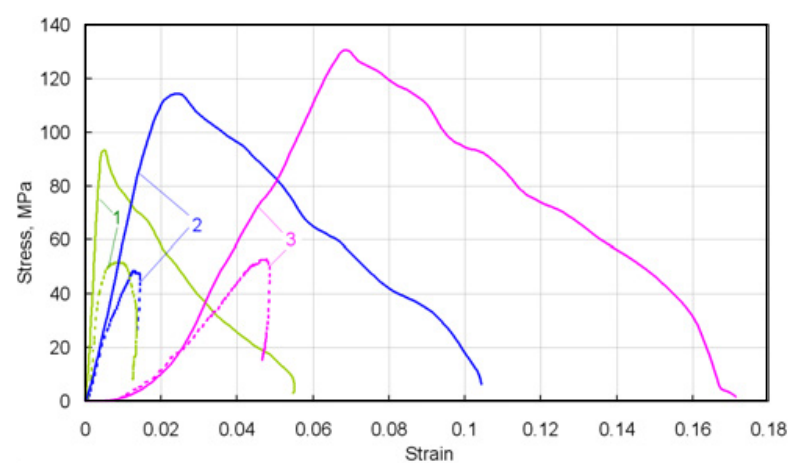

Fig. 11. Behaviour of ZDC No2 at uniaxial stress for different length of specimen: $1-20 \mathrm{~mm}, 2-10 \mathrm{~mm}, 3-5 \mathrm{~mm}$.

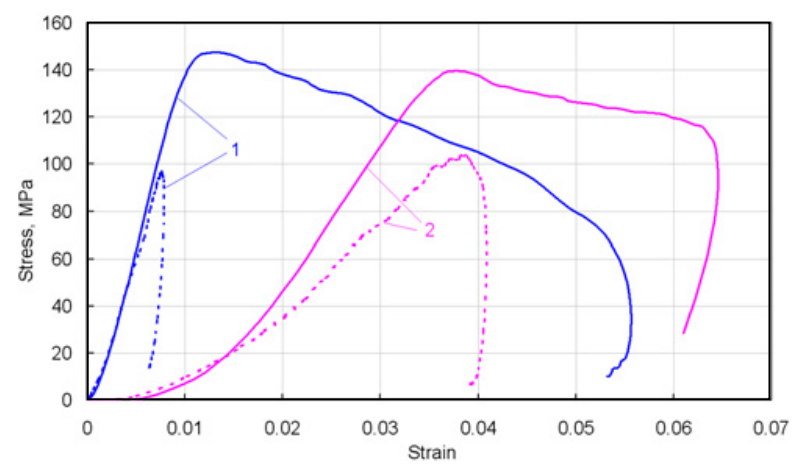

Fig. 12. Behaviour of ZDC No3 at uniaxial stress for different length of specimen: $1-10 \mathrm{~mm}, 2-5 \mathrm{~mm}$.

However the values of dynamic strength at compression practically do not depend on thickness of samples. Consequently the specific energy absorption of thin samples has appeared much more, than of thick. The more gentle slope of an active part of loading at the stress-strain curves, apparently, can be a consequence that the ceramics is a nonuniform granular material with the size of grains $\sim 1 \mathrm{~mm}$, and the general contribution of equability of surface of a sample into its deformability is more significant for thin samples by thickness $2-5 \mathrm{~mm}$.

The strength properties of ceramics and zirconium alumina concrete experimentally can be useful to verify a theoretical description of behaviour of those structural and protective building materials subjected to static and dynamic loads.

Growth rate of stress exert essential influence on strengthening properties of ceramics and concrete. The ceramics of batch No3 has the highest strength and smaller deformability. The ceramics is rather inert, and its temperature of melting is approximately equal $3000 \mathrm{~K}$. Heat conductivity of materials on a basis of zirconium dioxide in 1,5-2 times lower, than materials on the basis of others high refractory oxides. Thus, the ceramics on a basis of zirconium dioxide is the most suitable material for trap manufacturing. Its use in designs of traps will allow to raise considerably reliability of the mentioned devices and so to solve the basic problem of maintenance of safety of the atomic power station. 


\section{Acknowledgements}

The work was partially supported by the Russian Foundation for Basic Research (grant 10-01-00585-a).

\section{References}

1. E.D.H. Davies, S.C. Hunter, J. Mech. Phys. Solids. 11 155 (1963)
2. A.M. Bragov, A.K. Lomunov, Int. J. Impact Engineering, 16 (2) 321 (1995)

3. A.M. Bragov, G.M. Grushevsky, A.K. Lomunov, Exper. Mechanics 36(3) 237 (1996)

4. A.M. Bragov, G.M. Grushevsky, A.K. Lomunov, DYMAT J. 1253 (1994)

5. J. Rodriguez, C. Navarro, V. Sánchez-Gálvez, J. Phys. IV France, 04101 (1994)

6. K. Terzaghi Theoretical soil mechanics, Wiley, New York (1943) 